\title{
Immunophenotyping by Flow Cytometry of Fine Needle Aspirates in the Diagnosis of Lymphoproliferative Disorders: A Retrospective Study
}

\author{
Rui M.F. Henrique, ${ }^{1}$ M. Emília Sousa, ${ }^{2}$ M. Inês Godinho, ${ }^{2}$ Isabel Costa, ${ }^{1}$ \\ Isabel L. Barbosa, ${ }^{2 \star}$ and Carlos A.S. Lopes ${ }^{1}$ \\ ${ }^{1}$ Department of Pathology, The Portuguese Cancer Institute-Porto Regional Center, \\ Porto, Portugal \\ ${ }^{2}$ Laboratory of Cellular Immunology, Blood Bank, The Portuguese Cancer Institute-Porto Regional Center, \\ Porto, Portugal
}

\begin{abstract}
In order to determine the value of flow cytometric (FCM) immunophenotyping of fine-needle aspirates (FNA) in the diagnosis and classification of lymphoproliferative diseases, 61 tissue samples were studied and compared with the cytologic/histological results. In vivo and ex vivo FNA biopsy yielded the material for FCM, which comprised an extensive number of lymphoid cell markers. In all but three cases sufficient cells were collected. Overall, malignancy was diagnosed in 33 cases from a total of $47(70.2 \%)$, and in the remaining cases malignancy was not detected. Eleven cases were correctly diagnosed as reactive pro-
\end{abstract}

cesses (11/11). There were no false positive cases of malignancy, as diagnosed by FCM-FNA. The best accuracy was achieved in the low-grade B-cell lymphomas and lymphoblastic lymphoma/leukemia. We conclude that in a significant number of cases, FCM-FNA permits the separation between lymphoid malignancies and reactive processes without false positive results. It was found to be particularly useful in the differential diagnosis of mantle-cell and smalllymphocytic lymphoma and in the identification of lymphoblastic lymphoma/leukemia. J. Clin. Lab. Anal. 13:224-228, 1999. (C) 1999 Wiley-Liss, Inc.

Key words: lymphoproliferative diseases; fine-needle aspiration biopsy; surface markers; flow cytometry; immunophenotyping; lymphoma

\section{INTRODUCTION}

The role of fine-needle aspiration biopsy (FNA) in the diagnosis and staging of lymphoid malignancies is still a matter of debate (1-4). The main concerns in this regard rely on its accuracy, reliability, and usefulness when compared with the standard diagnostic tool, i.e., surgical biopsy (5-7). The use of ancillary techniques, such as immunophenotyping and flow cytometric analysis (FCM), provides an important diagnostic adjunct to the diagnosis of these diseases $(5,7-10)$.

The most recent system for lymphoma categorisationthe REAL classification - relies heavily on both morphological and immunophenotypical data (11). However, there are some antibodies that are not available for use on paraffin sections (12) or that use is subject to some technical refinements, some of them quite expensive, such as the use of a tyramide detection system. Because FCM can provide a wider range of markers to be tested and allows, in a relatively easy way, its quantitation and the simultaneous analysis of two or more markers, it could be envisaged as a potentially powerful tool in the definition of some lymphoproliferative diseases (LPD), either in conjunction with FNA or biopsy specimens $(5,7,9,13)$.

Thus, we undertook a retrospective study in order to evaluate the usefulness and potential limitations of FCM in conjunction with FNA, in the diagnosis of LPD in a relatively large group of these diseases. For that purpose, the data obtained by FCM (either in vivo or ex vivo), was compared with the morphological and immunohistochemical features of the respective cytological smear and surgical biopsy specimen. In contrast to previous studies, a uniform classification of the lymphoid malignancies was used both for the diagnoses performed by histology and for FCM-FCA, i.e., the REAL classification (11). Therefore, the true usefulness of

*Correspondence to: Isabel L. Barbosa, Ph.D., Serviço de ImunoHemoterapia, Instituto Português de Oncologia de Francisco GentilCentro Regional do Porto, Rua Dr. António Bernardino Almeida, 4200 Porto, Portugal.

Received 8 March 1999; Accepted 16 May 1999 
FCM-FNA in the categorisation of the non-Hodgkin's lymphomas could be thoroughly evaluated. Additionally, the inclusion of reactive lesions allowed us to determine the discriminative power of FCM-FNA in the differential diagnosis between lymphoid neoplasia and hiperplasia.

\section{MATERIALS AND METHODS}

\section{Sampling Collection and Fine-Needle Aspiration}

This study was comprised of 61 patients with suspected lymphoproliferative disease, observed in our institution between June, 1996, and November, 1998, and FNA was performed, either in vivo or ex vivo, in all cases. In the latter case, the lymph nodes were submitted fresh to our department of Pathology. A 23-gauge needle attached to a 10 -ml syringe was used, with the aid of an aspiration gun in the in vivo FNAs. In some cases, the specimens were obtained by computed tomography (CT) guidance, allowing the performance of the histological biopsy, as well. A drop of the aspirate was expressed onto glass slides, which were subsequently air-dried and stained using the DiffQuik method, or were alcohol-fixed and stained using a Papanicolaou stain. Then, each aspirate was flushed into $1.5 \mathrm{ml}$ of phosphate-buffered saline (PBS) and immediately sent to the flow cytometry laboratory.

The diagnoses performed on the smears were classified as small cell lymphoma, large cell lymphoma, lymphoblastic lymphoma/leukemia, Burkitt's lymphoma, reactive process, and nondiagnostic.

\section{Morphology and Histology}

Surgical biopsy samples were obtained in all 61 cases, either $\mathrm{CT}$ guided or by direct surgical excision. The tissue specimens were formalin fixed and processed for paraffin inclusion. Routine histologic sections and immunostains (using the avidinbiotin peroxidase technique) allowed the classification of each case according to the REAL classification of lymphoid neoplasms (11). The diagnoses performed were then placed in one of seven categories for purposes of analysis only: low-grade Bcell lymphomas (LGBL), high-grade B-cell lymphomas (HGBL), high-grade T-cell lymphomas (HGTL), lymphoblastic lymphoma/leukemia (ALL), miscellaneous (M), reactive process (RP), and nondiagnostic (ND). These diagnoses were made without the aid of the FCM results.

Hodgkin's disease cases were excluded from this study.

\section{Preparation of FNA Samples for Flow Cytometry}

All the samples were processed and analysed within 24 hours of collection. Prior to labelling the majority of the samples required concentration. The samples were transferred into Hanks' balanced salt solution (HBSS), centrifuged at 1100 $\mathrm{rpm}$ for $10 \mathrm{~min}$, and resuspended in approximately $500 \mu \mathrm{l}$. Leucocyte concentration was determined either by crystal violet enumeration or using a haematologic counter and adjusted to $10^{6}$ cells $/ \mathrm{ml}$.

\section{Monoclonal Antibody Panel and Flow Cytometry Analysis}

A minimum of $10^{5}$ cells per tube were used to perform the monoclonal antibody staining. Double-labelled fluorescent monoclonal antibodies (McAb) CD45/CD14, HLA-DR/CD3, CD5/CD19, kappa/lambda, CD4/CD8, and single-labelled McAb CD10, CD20, CD23, and TdT (all McAbs were from Dako Labometer, Portugal).

FNA samples were labelled with McAb for $15 \mathrm{~min}$ at room temperature followed by a 10-min incubation with $2.0 \mathrm{ml}$ of 1X FACS Lysing solution (BD-ENZifarma Lisboa, Portugal) for red cell lysis. After centrifugation at $1100 \mathrm{rpm}$ for $10 \mathrm{~min}$, the stained cells were resuspended in approximately $500 \mu \mathrm{l}$ of CellFix ${ }^{\mathrm{TM}}$ (BD-ENZifarma Lisboa). CD45 was used as positive control and double-conjugated mouse immunoglobulin $\mathrm{G}(\mathrm{IgG})$ was used as a negative control.

Using a FACSort flow cytometer and the LYSIS II software (BD-ENZifarma Lisboa), a two-colour, four-parameter flow cytometry analysis was performed and a minimum of 10,000 events were acquired for each sample. The results were expressed as the percentage of positive cells of the total nucleated cell population.

A pattern of "normality" was established based on the presence of a mixture of T- and B-cells without evidence of monoclonality or aberrant immunophenotype. B-cell malignancies were suggested when the immunoglobulin light-chain ratio was equal or greater than 6:1. Abnormal patterns of CD4 and CD8 expression (e.g., coexpression of both these antigens, lack of expression of both of them, or altered CD4/ CD8 ratio) or lack of expected T-cell markers (e.g., CD2) was considered diagnostic of T-cell neoplasia.

The FCM results were interpreted without knowledge of the FNA or surgical biopsy results. Then, the FCM results were combined with those of the FNA and a final diagnosis was achieved and placed in the same categories described for the histological specimens.

\section{Statistical Analysis}

To evaluate any differences between the groups of patients studied we used the $t$-test for Independent Samples, performed in a statistic software. We accepted results with a $P$-level $<0.05$ as "statistically significant" and those with a $P$-level $<0.005$ as "highly significant."

\section{RESULTS}

\section{Morphologic Categories}

In a total of 61 patients, a histological diagnosis was reached in 58 cases (95\%) and the results are summarised in Table 1. In the remainder the sample was insufficient 
TABLE 1. Detection Rate Flow Cytometric Immunophenotyping in Relation to the Histologic Categories of Lymphoproliferative Diseases

\begin{tabular}{lc} 
Histologic category & Detected by FCM \\
\hline Low-grade B-cell lymphoma & $15 / 17$ \\
Follicular centre-cell lymphoma & $3 / 5$ \\
Mantle-cell lymphoma & $8 / 8$ \\
Small-lymphocytic lymphoma/chronic & $4 / 4$ \\
$\quad$ lymphocytic leukemia & \\
High-grade B-cell lymphoma & $9 / 15$ \\
Diffuse large B-cell lymphoma & $6 / 12$ \\
Burkitt's lymphoma & $3 / 3$ \\
High-grade T-cell lymphoma & $0 / 4$ \\
Lymphoblastic lymphoma/leukemia & $9 / 9$ \\
Miscellaneous & $0 / 2$ \\
T-cell rich B-cell lymphoma & $0 / 1$ \\
Dermatopathetic lymphadenopathy in mycosis & $0 / 1$ \\
$\quad$ fungoides (LN2) & \\
Reactive process & $11 / 11$ \\
Nondiagnostic & $3 / 3$ \\
\hline
\end{tabular}

(two cases) or necrotic (one case), allowing no definitive diagnosis (ND category). There were 17 cases of LGBL: 5 follicular centre cell lymphoma (FCCL), 8 mantle-cell lymphoma (MCL), and 4 small lymphocytic lymphoma/ chronic lymphocytic leukemia (SLL/CLL). The LGBL cases corresponded to the small-cell lymphoma cases diagnosed by FNA. The HGBL group was comprised of 15 cases: 12 diffuse large B-cell lymphoma (DLBL) and 3 Burkitt's lymphoma (BL). Four anaplastic large-cell lymphoma (ALCL) composed the group of HGTL. The HGBL and HGTL cases were diagnosed cytologically as largecell lymphomas. The BL cases were all identified cytologically, as well. Concerning the ALL group, there were 9 cases: 5 B-cell and 4 T-cell phenotype. These cases were also correctly identified in the cytological smears. The miscellaneous category included one case of T-cell rich B-cell lymphoma and one case of dermatopathic lymphadenopathy, in a context of mycosis fungoides (LN2). These cases were not diagnosed as such by FNA and were inter- preted as reactive processes. Eleven cases were considered reactive (RP), without evidence of lymphoid malignancy.

\section{Immunological Phenotyping of FNA Samples by Flow Cytometry}

From a total of 61 FNA, only in 3 cases was the number of cells collected insufficient to stain $\left(<5 \times 10^{5}\right.$ cells $/ \mathrm{ml}$ in a total volume of $<1.5 \mathrm{ml}$ and these were the same cases in which histological and cytological diagnosis was not achieved). In this retrospective study, it was found that a minimum of $1 \times 10^{6}$ cells was required to perform the phenotypic analysis.

Lymphoid malignancy was diagnosed by FCM in 33 of the 47 pathologic cases identified by histology (70.2\%), based on the criteria previously cited (Table 1). When FCM results were combined with the FNA results, these cases corresponded to all the ALL (9/9), 88\% of the LGBL (15/17), and $60 \%$ of the HGBL (9/15).

The results of the flow cytometric immunophenotyping of the 58 cases analysed are displayed on Table 2 . In relation to the ALL group, the results obtained by FCM-FNA were consistent with those obtained by histologic analysis, in terms of the phenotype, and all the cases were TdT positive.

The comparison between LGBL and HGBL showed that the latter subgroup had a higher proportion of T cells (16 vs. $69 \%, P=0.002)$. In contrast, LGBL cases had more B-cells with a predominance of immunoglobulin kappa chain expression ( 86 vs. $14 \%, P<0.02$ ). In HGBL the proportion of neoplasms expressing immunoglobulin kappa or lambda light chains was equivalent.

Concerning the LGBL group, 12 cases were found to be CD5+. The CD23 expression allowed their classification as MCL (CD5+ and CD23-) or SLL/CLL (CD5+ and CD23+). The remainders were $\mathrm{CD} 10+, \mathrm{CD} 5-$; and CD23-, and were classified as FCCL, in conjunction with the cytological observation.

For the other 25 samples the FCM immunological phenotyping was not able to find evidence of lymphoid ma-

TABLE 2. Frequency of Immunophenotypical Markers in the Various Diagnostic Groups by Flow Cytometry Analysis ${ }^{\text {a }}$

\begin{tabular}{|c|c|c|c|c|}
\hline & CD3 & CD19/20 & $\kappa / \lambda$ ratio & Other markers \\
\hline Low-grade B-cell lymphoma & + & +++ & $+++/-$ & \\
\hline Follicular centre-cell lymphoma & & & & CD5-CD23-, CD10+ \\
\hline Mantle-cell lymphoma & & & & CD5+, CD23- \\
\hline Small-lymphocytic lymphoma/chronic lymphocytic leukemia & & & & CD5+, CD23+ \\
\hline High-grade B-cell lymphoma & ++ & + & $+/+$ & \\
\hline Diffuse large B-cell lymphoma & ++ & + & $+/+$ & \\
\hline Burkitt's lymphoma & + & ++ & & TdT-, CD10+ \\
\hline High-grade T-cell lymphoma & ++ & + & & \\
\hline \multicolumn{5}{|l|}{ Lymphoblastic lymphoma/leukemia } \\
\hline B-cell & - & +++ & & TdT+++, CD10+++, HLA-DR+++ \\
\hline T-cell & ++ & - & & TdT++ \\
\hline Reactive process & ++ & + & & \\
\hline
\end{tabular}

${ }^{\mathrm{a}}$ Data are expressed as $\%$ of positive cells and classified as follows: $+++\geq 75 \% ; 50 \% \leq++<75 \% ; 20 \% \leq+<50 \% ;-<20 \%$. 
lignancy. In the four HGTL and two miscellaneous cases, although FCM showed a predominance of $\mathrm{T}$ lymphocytes, a diagnosis was not established because there was not sufficient evidence of abnormal or aberrant phenotype. In eight cases of B-cell lymphoma, there was no evidence of monoclonality by FCM. The remaining eleven cases classified by histology as RP cases showed a normal pattern of Tand B-cell populations.

\section{DISCUSSION}

Several previous studies have addressed the topic of the usefulness and limitations of FNA alone or in conjunction with FCM in the diagnosis and classification of LPD $(1,7,8,10)$. However, to our knowledge, this is the first study to investigate this problem in the context of the REAL classification scheme.

In our study, LGBL was the most reliably identifiable group of LPD by means of FCM-FNA. Additionally, it was possible to diagnose, with a great degree of certainty, the MCL and the SLL/CLL subgroups, among the others. This is a major point in favour of FCM, since the morphological differential diagnosis between these two entities is not very easy in some instances (14). Although the overexpression of the $\mathrm{Bcl}-1$ gene product (i.e., cyclin D1) is claimed to be specific for the diagnosis of MCL $(15,16)$, its detection by immunohistochemical methods in paraffin sections is somewhat difficult and may need expensive enhancement systems $(12,17)$. At present, this distinction may not carry therapeutical implications, but one should bear in mind that the prognosis of these two diseases is quite different $(11,14)$. In addition, if new therapeutic approaches are to be tested in MCL, it becomes crucial that the eligible cases should be correctly diagnosed.

Regarding DLBL, the rate of correctly identified cases was somewhat inferior to the previously discussed LGBL (50 vs. $88 \%$ ), but is similar to that of other studies (7). Two reasons can be pointed out to justify this discrepancy. The first is that in DLBL, the number of reactive cells (especially T-lymphocytes) is higher than in LGBL. This may influence the diagnostic accuracy in two ways: the mixture of B- and T-cells raises the problem of a reactive LPD, and the lower number of neoplastic cells may jeopardise the FCM analysis for monoclonality. The second reason for less accuracy in the detection of DLBL was the partial involvement of the lymph node (as observed in the surgical biopsy specimen) which further impairs obtaining neoplastic cells for FCM. It is noteworthy, in this regard, that these same problems may affect the cytological assessment, and therefore may raise some concern on the accuracy of the negative, i.e., nonneoplastic cases as diagnosed by FCM-FNA.

FCM correctly identified all cases of BL as B-cell malignancies. Their categorisation as a specific entity was made with the aid of the cytological observation which allowed the identification of the numerous cytoplasmic (lipid) vacuoles, typical of this lymphoma (18). However, without the cytological assessment, the diagnosis by FCM alone would not be possible.

A similar problem to that of DLBL arose in the diagnosis of HGTL, which were all of the anaplastic large-cell type. Indeed, the recognition of T-cell malignancies is more difficult than their B-cell counterparts, because one must rely on the detection of an aberrant pattern of T-cell antigen expression or the abnormal distribution of T-cell antigens (19). In our cases, another problem was raised: the considerable number of reactive cells that may be present in the context of ALCL (20,21). However, the sample size was rather small (just four cases) and it was not representative of most T-cell lymphomas, which seem to be diagnosed by these means with more accuracy than in our study $(7,9)$. Perhaps the use of a monoclonal antibody against CD30 could provide more accurate diagnosis in our cases, as it does in the context of Hodgkin's disease (9).

The problems of cell sampling and number were also determinant for the nonrecognition of the cases of T-cell rich Bcell lymphoma and of dermatopathic lymphadenopathy, in a context of mycosis fungoides (LN2). We could confirm this same problem in the biopsy specimen, because the neoplastic cells were quite difficult to find in the reactive background. The difficulty in diagnosing a T-cell rich B-cell lymphoma in FNA with FCM was already highlighted by other authors (22).

Concerning the ALL subgroup, FCM proved to be a very reliable ancillary technique, since all cases in this subgroup were shown to be TdT positive. Additionally, none of the cases of the other subgroups showed positivity for this marker. This result is quite important because it may preclude the need for surgical biopsy in the follow-up of these patients (most of them of a rather young age) whenever a lymph node or extranodal relapse is suspected. This finding may also be important in the initial diagnosis, since the monoclonal antibodies against TdT for use in paraffin sections, in our experience, do not always provide reliable results. Additionally, FCM permits the investigation of HLA-DR expression and the presence or absence of surface immunoglobulins with great confidence. Because these markers are difficult to characterise on paraffin sections, their study by FCM may be very helpful in suspecting and/or establishing a diagnosis in dubious cases.

Another major result of our study was the absence of falsepositive results on FCM-FNA: none of the reactive cases were interpreted in FCM as a B-cell or T-cell malignancy. However, as previously stated, a significant number of nonHodgkin's lymphomas were also diagnosed as "without evidence of malignancy" by FCM. This may be partially explained by the strict criteria used for the definition of Band T-cell neoplasms. In these cases, either the cytological assessment or the persistence of a clinical suspicion was determinant for the diagnosis, through the performance of a surgical biopsy.

In summary, our results demonstrate that FCM-FNA pro- 
vides an important aid to the distinction between malignant and nonmalignant LPD in most cases. A negative result ("without evidence of malignancy") must always be regarded with great care and the need for a surgical biopsy should be addressed on a clinical suspicion basis. In the cases of lymphoid malignancy, this method is particularly relevant in the low-grade B-cell lymphomas, allowing some very important differential diagnosis in difficult cases. Additionally, it may provide a very reliable and useful approach in cases of suspected disease relapse in the ALL subgroup.

\section{ACKNOWLEDGMENTS}

The authors greatly appreciate the excellent technical assistance of Ms. Angela Silvestre and Ms. Paula Lopes in the performance of the immunostains in paraffin sections, and of Ms. Rosa M. Moreira in the preparation of some of the samples for flow cytometry analysis.

\section{REFERENCES}

1. Carter TR, Feldman PS, Innes DJ, Frierson HF, Frigy AF. The role of fine needle aspiration in the diagnosis of lymphoma. Acta Cytol 1988;32:848-853.

2. Frable WJ, Kardos TF. Fine needle aspiration biopsy. Applications in the diagnosis of lymphoproliferative diseases. Am J Surg Pathol 1988;12:62-72.

3. Kardos TF, Maygarden SJ, Blumberg AK, Wakely PE, Frable WJ. Fine needle aspiration biopsy in the management of children and young adults with peripheral lymphadenopathy. Cancer 1989;63:703-707.

4. Moriarty AT, Banks ER, Bloch T. Cytologic criteria for subclassification of Hodgkin's disease using fine-needle aspiration. Diagn Cytopathol 1989;5:122-125.

5. Dunphy $\mathrm{CH}$, Ramos R. Combining fine-needle aspiration and flow cytometric immunophenotyping in evaluation of nodal and extranodal sites for possible lymphoma: a retrospective review. Diagn Cytopathol 1997;16:200-206.

6. Ramzy I, Rone R, Schultenover SJ, Buhaug J. Lymph node aspiration biopsy: diagnostic reliability and limitations - an analysis of 350 cases. Diagn Cytopathol 1985;1:39-45.

7. Zander DS, Iturraspe JA, Everett ET, Massey JK, Braylan RC. Flow cytometry: in vitro assessment of its potential application for diagnosis and classification of lymphoid processes in cytologic preparations from fine-needle aspirates. Am J Clin Pathol 1994;101:577-586.
8. Robins DB, Katz RL, Swan F, Atkinson EN, Ordonez NG, Huh YO. Immunotyping of lymphoma by fine-needle aspiration. A comparative study of cytospin preparations and flow cytometry. Am J Clin Pathol 1994;101:569-576.

9. Saddik M, El Dabbagh L, Mourad WA. Ex vivo fine-needle aspiration cytology and flow cytometric phenotyping in the diagnosis of lymphoproliferative disorders: a proposed algorithm for maximun resource utilization. Diagn Cytopathol 1997;16:126-131.

10. Sneige N, Dekmezian R, El Naggar A, Manning J. Cytomorphologic, immunocytochemical and nucleic acid flow cytometric study of 50 lymph nodes by fine needle aspiration. Comparison with results obtained by subsequent excisional biopsy. Cancer 1991;67:1003-1007.

11. Harris NL, Jaffe ES, Stein H, et al. A revised European-American classification of lymphoid neoplasms: a proposal from the International Lymphoma Study group. Blood 1994;84:1361-1392.

12. Katz RL. Cytologic diagnosis of leukemia and lymphoma. Values and limitations. Clin Lab Med 1991;11:469-499.

13. Clatch RJ, Walloch JL. Multiparameter immunophenotyping analysis of fine-needle aspiration biopsies and other hematologic specimens by laser scanning cytometry. Acta Cytol 1997;41:109-122.

14. Medeiros LJ, Jaffe ES. Low-grade B-cell lymphomas not specified in the working formulation. In: Jaffe ES, editor. Surgical pathology of the lymph nodes and related organs. W.B. Saunders: Philadelphia; 1994. p 221-251.

15. Medeiros LJ, van Krieken J, Jaffe ES, Raffeld M. Association of Bcl-1 rearrangements with lymphocytic lymphoma of intermediate differentiation. Blood 1990;76:2086-2090.

16. Brynes RK, McCourty A, Tamayo R, Jenkins K, Battifora H. Demonstration of cyclin D1 (Bcl-1) in mantle cell lymphoma. Enhanced staining using heat and ultrasound epitope retrieval. Appl Immunohistochem 1997;5:45-50.

17. Zuckerberg LR, Yang W-I, Arnold A, Harris NL. Cyclin D1 expression in non-Hodgkin's lymphomas. Detection by immunohistochemistry. Am J Clin Pathol 1995;103:756-760.

18. Ferry JA, Harris NL, editors. Atlas of lymphoid hyperplasia and lymphoma. W.B. Saunders: Philadelphia; 1997.

19. Picker LJ, Weiss LM, Medeiros LJ, Wood GS, Warnke RA. Immunophenotypic criteria for the diagnosis of non-Hodgkin's lymphoma. Am J Pathol 1987;128:181-201.

20. Agnarsson B, Kadin M: Ki-1 positive large cell lymphoma: a morphologic and immunologic study of 19 cases. Am J Surg Pathol 1988;12:264-274.

21. Kadin ME: Ki-1-positive anaplastic large cell lymphoma: a distinct clinicopathologic entity. Ann Oncol 1994;5(Suppl.1):S25-S30.

22. Galindo LM, Havlioglu N, Grosso LE. Cytologic findings in a case of T-cell rich B-cell lymphoma: potential diagnostic pitfalls in FNA of lymph nodes. Diagn Cytopathol 1996;14:253-258. 